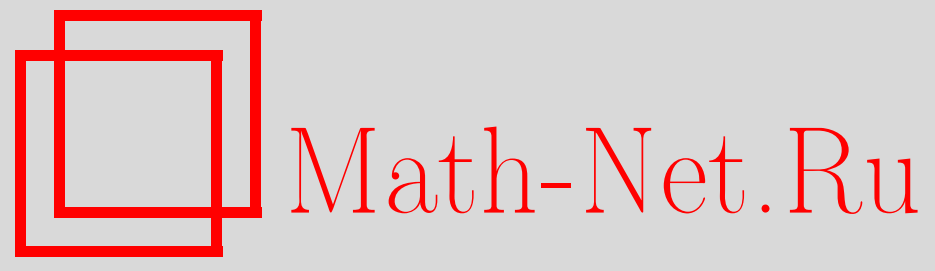

Б. О. Волков, Даламбертианы Леви и их применение в квантовой теории, Вестн. Сам. гос. техн. ун-та. Сер. Физ.-мат. науки, 2015, номер 2, 241258

DOI: https://doi.org/10.14498/vsgtu1372

Использование Общероссийского математического портала MathNet.Ru подразумевает, что вы прочитали и согласны с пользовательским соглашением

http: //www.mathnet.ru/rus/agreement

Параметры загрузки:

IP : 3.81 .55 .215

26 апреля 2023 г., 06:24:58

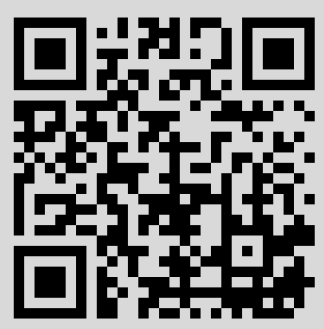




\title{
ДАЛАМБЕРТИАНЫ ЛЕВИ И ИХ ПРИМЕНЕНИЕ В КВАНТОВОЙ ТЕОРИИ*
}

\section{Б. О. Волков}

Московский государственный технический университет им. Н. Э. Баумана, Россия, 105005, Москва, 2-я Бауманская ул., 5/1.

\begin{abstract}
Аннотация
Даламбертиан Леви - бесконечномерный дифференциальный оператор второго порядка, определенный по аналогии с лапласианом Леви. У работы две цели: исследовать связи между различными определениями даламбертиана Леви и исследовать связь между даламбертианами Леви и уравнениями квантовой хромодинамики (уравнениями Янга-Миллса-Дирака). Существуют два определения классического оператора Даламбера-Леви. Первое из них заключается в том, что этот оператор определяется как интегральный функционал, заданный специальным видом второй производной. По-другому даламбертиан Леви можно определить с помощью средних Чезаро вторых производных по направлению вдоль векторов ортонормированного базиса. В работе доказывается эквивалентность этих определений, при этом используются слабо равномерно плотные ортонормированные базисы. По аналогии с семейством неклассических лапласианов Леви в работе вводится семейство неклассических даламбертианов Леви, параметризованных линейными операторами на линейной оболочке базиса. Показано, что связь даламбертиана Леви с калибровочными полями можно описать как с помощью классического даламбертиана Леви, который задается тождественным оператором на линейной оболочке базиса, так и с помощью другого элемента семейства неклассических даламбертианов Леви. В работе изучается связь между последним оператором и уравнениями Янга-Милсса с источником. В частности, выводится система бесконечномерных уравнений, эквивалентная уравнениям квантовой хромодинамики и содержащая такой неклассический даламбертиан.
\end{abstract}

Ключевые слова: лапласиан Леви, даламбертиан Леви, уравнения Янга-Миллса, уравнения Янга-Миллса-Дирака.

doi: http://dx.doi.org/10.14498/vsgtu1372

(C) 2015 Самарский государственный технический университет.

\section{Образец для цитирования}

Волков Б. О. Даламбертианы Леви и их применение в квантовой теории // Becmн. Сам. гос. техн. ун-та. Сер. Физ.-мат. науки, 2015. Т. 19, № 2. С. 241-258. doi: $10.14498 /$ vsgtu1372.

\section{Сведения об авторе}

Борис Олегович Волков (к.ф.-м.н.; borisvolkov1986@gmail.com), доцент, каф. ФН-12«Математическое моделирование».

*Настоящая статья представляет собой расширенный вариант доклада [1], сделанного авторами на Четвёртой международной конференции «Математическая физика и её приложения» (Россия, Самара, 25 августа - 1 сентября 2014). 
Введение. В статье обсуждается связь между различными определениями даламбертиана Леви и выводится система бесконечномерных уравнений, содержащая даламбертиан типа Леви и эквивалентная уравнениям квантовой хромодинамики (уравнениям Янга-Миллса-Дирака).

Для функционалов, определенных на $L_{2}(0,1)$, Полем Леви были сформулированы несколько определений лапласиана Леви (см. [2]). Одно из определений состоит в следующем. Если $f$-функция на $L_{2}(0,1)$ такая, что для всех $x, u, v \in L_{2}(0,1)$ выполняется соотношение

$$
f^{\prime \prime}(x)(u, v)=\int_{0}^{1} \int_{0}^{1} K_{V}(x)(t, s) u(t) v(s) d t d t s+\int_{0}^{1} K_{L}(x)(t) u(t) v(t) d t,
$$

где $K_{V}(x) \in L_{2}([0,1] \times[0,1])$ и $K_{L}(x) \in L_{\infty}([0,1])$, то значение лапласиана Леви на функции $f$ определяется равенством

$$
\Delta_{L} f(x)=\int_{0}^{1} K_{L}(x)(t) d t .
$$

Другое определение состоит в следующем. Пусть $\left\{e_{n}\right\}-$ ортонормированный базис в $L_{2}(0,1)$. Тогда значение лапласиана Леви $\Delta_{L}$ на $f$ определяется равенством

$$
\Delta_{L} f(x)=\lim _{n \rightarrow \infty} \frac{1}{n} \sum_{k=1}^{n} f^{\prime \prime}(x)\left(e_{k}, e_{k}\right)
$$

(т.е. значение лапласиана Леви на функции $f$-это среднее Чезаро вторых производных этой функции по направлениям векторов из $\left.\left\{e_{n}\right\}\right)$. Если базис $\left\{e_{n}\right\}$-слабо равномерно плотный, эти два определения эквивалентны (см. $[2,3])$. Второе определение лапласиана Леви естественно переносится на пространство функций, определенных на оснащенном гильбертовом пространстве. Свойства такого оператора и его обобщений, так называемых неклассических и экзотических лапласианов Леви, изучались в [4-16] (см. также цитируемую в этих работах литературу).

Интерес к лапласиану Леви и его аналогам существенно возрос после того, как в $[17,18]$ Л. Аккарди, П. Джибилиско и И. В. Волович доказали, что связность в тривиальном векторном расслоении, базой которого является евклидово пространство, является решением уравнений Янга-Миллса тогда и только тогда, когда соответствующий связности параллельный перенос является Леви-гармоническим функционалом (см. также [19]). При этом использовался аналог первого определения лапласиана Леви, действующий на операторозначные функции, определенные на пространстве $C^{1}$-гладких кривых в евклидовом пространстве. Такой лапласиан задается более сложной формой второй производной, чем (1). Подход работ $[17,18]$ к лапласиану Леви был использован также в [20], где теорема о связи с уравнениями ЯнгаМиллса доказывалась в случае нетривиального векторного расслоения, базой которого является компактное риманово многообразие, для параллельного переноса вдоль $C^{1}$-гладких кривых и стохастического параллельного переноса. По аналогии с лапласианом Леви в $[17,18]$ был впервые введен даламбертиан Леви как интегральный функционал, определяемый специальным 
видом второй производной. Для даламбертиана Леви верна аналогичная теорема о связи с калибровочными полями на пространстве Минковского. В [5] и [14] были соответственно введены лапласиан и даламбертиан Леви на многообразиях, определенные с помощью среднего Чезаро вторых производных по направлению. Для таких операторов в [14] была доказана теорема о связи лапласиана Леви и даламбертиана Леви и калибровочных полей, при этом эквивалентность определений операторов с помощью чезаровских средних вторых производных по направлениям и определений с помощью интегрального представления не доказывалась. Для плоского случая эта эквивалентность доказывается ниже.

Статья устроена следующим образом. В первом параграфе по аналогии с семейством неклассических лапласианов Леви вводится семейство неклассических даламбертианов Леви. Во втором параграфе доказывается эквивалентность определения классического даламбертиана Леви с помощью чезаровских средних вторых производных по направлениям и определения с помощью интегрального представления. В третьем параграфе приводятся общие сведения о связи классического даламбертиана Леви и калибровочных полей, а также доказывается, что если параллельный перенос зависит от конечного количества векторов ортонормированного базиса, то соответствующая связность является плоской. В четвертом параграфе исследуется связь между неклассическим даламбертианом Леви и уравнениями Янга-Милсса с источником, а также выводится система бесконечномерных уравнений, эквивалентная уравнениям квантовой хромодинамики и содержащая такой даламбертиан и производную в конечной точке.

1. Лапласианы и даламбертианы Леви. Везде ниже, если $G_{1}, G_{2}$ - вещественные локально выпуклые пространства, для функции $f: G_{1} \rightarrow G_{2}$, дифференцируемой по Гато в точке $x$, символом $d_{u} f(x)$, где $u \in G_{1}$, обозначается

$$
\left.\frac{d}{d \varepsilon}\right|_{\varepsilon=0} f(x+\varepsilon u)=f^{\prime}(x) u .
$$

Напомним общую схему определения однородных линейных дифференциальных операторов первого и второго порядка из статьи [21], которая включает в себя лапласианы Гросса-Вольтерры и Леви. Пусть $E$ - вещественное локально выпуклое пространство и $E^{*}$ - его сопряженное пространство, наделенное *-слабой топологией. Пусть $S$ - линейный вещественный функционал, определенный на пространстве $\operatorname{dom} S \subset E^{*}$. Областью определения $\operatorname{dom} D_{S}$ дифференциального оператора первого порядка $D_{S}$, порожденного линейным функционалом $S$, является пространство всех дифференцируемых по Гато действительных функций на пространстве $E$, для которых $f^{\prime}(x) \in \operatorname{dom} S$ для каждого $x \in E$. Оператор $\operatorname{dom} D_{S}$ действует следующим образом:

$$
D_{S} f(x)=S\left(f^{\prime}(x)\right) \text { для } x \in E, f \in \operatorname{dom} D_{S} .
$$

Примером дифференциального оператора первого порядка является производная в конечной точке, определение которой приводится в четвертом параграфе.

Пусть $L\left(E, E^{*}\right)$ - пространство непрерывных линейных функционалов из $E$ в $E^{*}$ и пусть $S$ - линейный вещественный функционал, определенный на 
пространстве $\operatorname{dom} S \subset L\left(E, E^{*}\right)$. Областью определения $\operatorname{dom} D_{S}$ дифференциального оператора второго порядка $D_{S}$, порожденного линейным функционалом $S$, является пространство всех дважды дифференцируемых по Гато действительных функций на пространстве $E$, для которых $f^{\prime \prime}(x) \in \operatorname{dom} S$ для каждого $x \in E$. Оператор $\operatorname{dom} D_{S}$ действует следующим образом:

$$
D_{S} f(x)=S\left(f^{\prime \prime}(x)\right) \text { для } x \in E, f \in \operatorname{dom} D_{S} .
$$

Пусть $E$ непрерывно вложено в действительное сепарабельное гильбертово пространство $H$ так, что образ $E$ при вложении плотен в $H$. Тогда $E \subset H \subset E^{*}$ - оснащенное гильбертово пространство. Зафиксируем в $H$ ортонормированный базис $\left\{e_{n}\right\}$, состоящий из векторов пространства $E$. Обобщенный лапласиан Леви (или экзотический лапласиан Леви) $\Delta_{L}^{l}$ порядка $l \geqslant 0$-это дифференциальный оператор второго порядка, порожденный функционалом $\operatorname{tr}_{L}^{l}$ (экзотическим следом Леви), который определяется следующим образом:

$$
\operatorname{tr}_{L}^{l}(L)=\lim _{n \rightarrow \infty} \frac{1}{n^{l}} \sum_{k=1}^{n}\left(L e_{k}, e_{k}\right),
$$

а область его определения состоит из всех $L \in L\left(E, E^{*}\right)$, для которых правая часть (2) существует (см. [7, 10]). Определение экзотического лапласиана Леви при $l=0$ совпадает с определением лапласиана Гросса-Вольтерры, а при $l=1$ совпадает с определением классического лапласиана Леви. Пусть $R-$ линейный оператор, действующий из $\operatorname{span}\left\{e_{n}: n \in \mathbb{N}\right\}$ в $E$. Неклассический лапласиан Леви $\Delta_{L}^{R}$, порожденный оператором $R$, - это дифференциальный оператор второго порядка, порожденный линейным функционалом $\operatorname{tr}_{L}^{R}$, который определяется следующим образом:

$$
\operatorname{tr}_{L}^{R}(L)=\lim _{n \rightarrow \infty} \frac{1}{n} \sum_{k=1}^{n}\left(L R e_{k}, R e_{k}\right)
$$

а область его определения состоит из всех $L \in L\left(E, E^{*}\right)$, для которых правая часть (3) существует (см. [6]). Все экзотические лапласианы Леви, кроме лапласиана Гросса-Вольтерры, представляются как неклассические лапласианы Леви (см. [16], а также ср. [7,9]): для $l>-1$ выполняется равенство

$$
\Delta_{N^{-l / 2}}^{L}=(l+1) \Delta_{L}^{l+1}
$$

где оператор $N^{-l / 2}$ на $\operatorname{span}\left\{e_{n}: n \in \mathbb{N}\right\}$ определен следующим образом:

$$
N^{-l / 2} e_{n}=n^{-l / 2} e_{n}
$$

По аналогии можно определить даламбертиан Леви. Пусть теперь $E=$ $=\mathbb{R}^{1,3} \otimes E_{1}$ и $H=\mathbb{R}^{1,3} \otimes H_{1}$, где $E_{1}$ - вещественное локально выпуклое пространство, непрерывно вложенное в действительное сепарабельное гильбертово пространство $H_{1}$ так, что образ $E_{1}$ при вложении плотен в $H_{1}$. Пусть теперь $\left\{e_{n}\right\}$ - ортонормированный базис в $H_{1}$, состоящий из векторов пространства $E_{1}$. Пусть $\left\{p_{0}, p_{1}, p_{2}, p_{3}\right\}$ - базис в пространстве $\mathbb{R}^{1,3}=\left\{x_{0}, x_{1}, x_{2}, x_{3}\right\}$. 
Метрика $\eta$ в этом базисе имеет диагональный вид с диагональю $\{1,-1,-1,-1\}$. Неклассический даламбертиан Леви, порожденный линейным оператором $R: \operatorname{span}\left\{e_{n}: n \in \mathbb{N}\right\} \rightarrow E_{1}$, - это дифференциальный оператор второго порядка, порожденный линейным функционалом $S^{R}$, который определяется формулой

$$
\begin{aligned}
S^{R}(L)=\lim _{n \rightarrow \infty} \frac{1}{n}\left(\sum_{k=1}^{n}\left\langle L\left(p_{0} \otimes R e_{k}\right), p_{0} \otimes R e_{k}\right\rangle-\right. & \\
& \left.-\sum_{\mu=1}^{3}\left\langle L\left(p_{\mu} \otimes R e_{k}\right), p_{\mu} \otimes R e_{k}\right\rangle\right),
\end{aligned}
$$

а область его определения состоит из всех $L \in L\left(E, E^{*}\right)$, для которых правая часть (4) существует. По-другому определение неклассического даламбертиана Леви можно сформулировать следующим образом.

ОПРЕДЕЛЕНИЕ 1. Неклассическим даламбертианом Леви, порожденным оператором $R$, называется отображение из Dom $\square_{R}^{L}$ в пространство функций на $E$, определенное формулой

$$
\square_{L}^{R} f(x)=\lim _{n \rightarrow \infty} \frac{1}{n} \sum_{k=1}^{n}\left(d_{p_{0} \otimes R e_{k}} d_{p_{0} \otimes R e_{k}} f(x)-\sum_{\mu=1}^{3} d_{p_{\mu} \otimes R e_{k}} d_{p_{\mu} \otimes R e_{k}} f(x)\right),
$$

где $\operatorname{Dom} \square_{R}^{L}$ - пространство дважды дифференцируемых по Гато функций на $E$, для которых правая часть (5) существует для всех $x \in E$.

Если $R$ - тождественный оператор, то порожденный $R$ даламбертиан Леви - классический даламбертиан Леви.

2. Даламбертиан Леви, определенный с помощью интегрального представления. Греческие индексы пробегают $\{0,1,2,3\}$, если не оговорено иное. Мы будем суммировать по повторяющимся индексам. Символ $M_{N}(\mathbb{C})$ обозначает пространство квадратных комплексных матриц порядка $N$.

Напомним определение из [17]. Обозначим символом $\Omega$ пространство дважды дифференцируемых по Фреше функций из $C^{1}\left([0,1], \mathbb{R}^{1,3}\right)$ в $M_{N}(\mathbb{C})$, для второй производной которых в каждой точке $\sigma \in C^{1}\left([0,1], \mathbb{R}^{1,3}\right)$ выполняется соотношение

$$
\begin{aligned}
& f^{\prime \prime}(\sigma)(u, v)=\int_{0}^{1} \int_{0}^{1} K_{\mu \nu}^{V}(\sigma)(t, s) u^{\mu}(t) v^{\nu}(s) d t d s+ \\
& \quad+\int_{0}^{1} K_{\mu \nu}^{L}(\sigma)(t) u^{\mu}(t) v^{\nu}(t) d t+\int_{0}^{1} K_{\mu \nu}^{S}(\sigma)(t)\left(\dot{u}^{\mu}(t) v^{\nu}(t)+\dot{v}^{\mu}(t) u^{\nu}(t)\right) d t
\end{aligned}
$$

где $u, v \in C^{1}\left([0,1], \mathbb{R}^{1,3}\right)$ такие, что

$$
u(0)=v(0)=u(1)=v(1)=0,
$$

$K_{\mu \nu}^{V} \in L_{2}\left([0,1] \times[0,1], M_{N}(\mathbb{C})\right), K_{\mu \nu}^{L}, K_{\mu \nu}^{S} \in L_{\infty}\left([0,1], M_{N}(\mathbb{C})\right), K_{\mu \nu}^{L}$ - симметричный тензор:

$$
K_{\mu \nu}^{L}=K_{\nu \mu}^{L}
$$


а $K_{\mu \nu}^{S}$ - антисимметричный тензор:

$$
K_{\mu \nu}^{S}=-K_{\nu \mu}^{S}
$$

ОПРеДЕЛЕниЕ 2. Даламбертиан Леви-это линейное отображение из $\Omega$ в пространство всех $M_{N}(\mathbb{C})$-значных функций на $C^{1}\left([0,1], \mathbb{R}^{1,3}\right)$, определенное формулой

$$
\square_{L_{1}} f(\sigma)=\int_{0}^{1} \eta^{\mu \nu} K_{\mu \nu}^{L}(\sigma)(t) d t
$$

Напомним определение из [2].

ОПРеДЕЛЕниЕ 3. Ортонормированный базис $\left\{h_{n}\right\}$ в $L_{2}(0,1)$ называется слабо равномерно плотным, если

$$
\lim _{n \rightarrow \infty} \int_{0}^{1} h(t)\left(\frac{1}{n} \sum_{k=1}^{n} h_{k}^{2}(t)-1\right) d t=0
$$

для любой функции $h \in L_{\infty}[0,1]$.

Примером слабо равномерно плотного базиса в $L_{2}(0,1)$ является последовательность $h_{n}(t)=\sqrt{2} \sin n \pi t$.

Следующая теорема связывает определения 1 и 2.

Теорема 1. Пусть $\left\{h_{n}\right\}$ - слабо равномерно плотный базис в $L_{2}(0,1)$, состоящий из функицй из $C^{1}([0,1], \mathbb{R})$ таких, что $h_{n}(0)=h_{n}(1)=0$ для всех $n \in \mathbb{N}$. Если $f \in \Omega$, mo

$$
\square_{L_{1}} f(\sigma)=\lim _{n \rightarrow \infty} \frac{1}{n} \sum_{k=1}^{n}\left(d_{p_{0} h_{k}} d_{p_{0} h_{k}} f(\sigma)-\sum_{\mu=1}^{3} d_{p_{\mu} h_{k}} d_{p_{\mu} h_{k}} f(\sigma)\right) .
$$

До ка з а тель ст в о. Действительно, т.к. $K_{\mu \nu}^{S}-$ антисимметричный тензор и $h_{k}(0)=h_{k}(1)=0$, выполняется равенство

$$
f^{\prime \prime}(\sigma)\left(p_{\mu} h_{k}, p_{\mu} h_{k}\right)=\int_{0}^{1} \int_{0}^{1} K_{\mu \mu}^{V}(\sigma)(t, s) h_{k}(t) h_{k}(s) d t d s+\int_{0}^{1} K_{\mu \mu}^{L}(\sigma)(t) h_{k}^{2}(t) d t .
$$

Так как $\left\{h_{n}\right\}$-ортонормированный базис в $L_{2}(0,1)$ и $K_{\mu \mu}^{V}(\sigma) \in L_{2}([0,1] \times$ $\times[0,1])$, выполняется равенство

$$
\lim _{n \rightarrow \infty} \int_{0}^{1} \int_{0}^{1} K_{\mu \mu}^{V}(\sigma)(t, s) h_{n}(t) h_{n}(s) d t d s=0
$$

Так как $\left\{h_{n}\right\}$ - слабо равномерно плотный базис и $K_{\mu \mu}^{V}(\sigma) \in L_{\infty}([0,1])$, выполняется равенство

$$
\lim _{n \rightarrow \infty} \frac{1}{n} \sum_{k=1}^{n} \int_{0}^{1} K_{\mu \mu}^{L}(\sigma)(t) h_{k}^{2}(t) d t=\int_{0}^{1} K_{\mu \mu}^{L}(\sigma)(t) d t
$$


Отсюда следует утверждение теоремы.

ЗАмечание 1. Определение лапласиана Леви с помощью интегрального представления получается, если в определении 2 метрику Минковского заменить на евклидову. Утверждение, аналогичное утверждению теоремы 2, верно для лапласиана Леви.

3. Даламбертиан Леви и уравнения Янга-Миллса. Ниже связность в тривиальном векторном расслоении с базой $\mathbb{R}^{1,3}$, слоем $\mathbb{C}^{N}$, структурной группой $U(N)$, ассоциированном с тривиальным главным расслоением $U(N) \times \mathbb{R}^{1,3}$, задана на $\mathbb{R}^{1,3}$ как $u(N)$-значная $C^{\infty}$-гладкая 1 -форма $A_{\mu}(x) d x^{\mu}$, определенная на $\mathbb{R}^{1,3}$. Для функции $\varphi \in C^{\infty}\left(\mathbb{R}^{1,3}, u(N)\right)$ ковариантная производная вдоль поля $\left(\partial / \partial_{\mu}\right)$ определяется так:

$$
\nabla_{\mu} \varphi=\partial_{\mu} \varphi+\left[A_{\mu}, \varphi\right]
$$

Тензор кривизны определяется формулой

$$
F_{\mu \nu}=\partial_{\mu} A_{\nu}-\partial_{\nu} A_{\mu}+\left[A_{\mu}, A_{\nu}\right]
$$

Для кривой $\sigma \in W_{2}^{1}\left([0,1], \mathbb{R}^{1,3}\right)$ оператор $U_{t, s}(\sigma)$, где $0 \leqslant s \leqslant t \leqslant 1$, определяется как решение системы дифференциальных уравнений ${ }^{1}$

$$
\left\{\begin{array}{r}
\frac{d}{d t} U_{t, s}(\sigma)=-A_{\mu}(\sigma(t)) \dot{\sigma}^{\mu}(t) U_{t, s}(\sigma) \\
\frac{d}{d s} U_{t, s}(\sigma)=U_{t, s}(\sigma) A_{\mu}(\sigma(s)) \dot{\sigma}^{\mu}(s) \\
\left.U_{t, s}(\sigma)\right|_{t=s}=I_{N} .
\end{array}\right.
$$

Решение уравнения (7) выражается в виде ряда

$$
U_{t, s}(\sigma)=I_{N}+\sum_{k=1}^{\infty} \int_{\Delta_{s, t}^{k}} d \tau_{1} \ldots d \tau_{k}\left(-A_{\mu}\left(\sigma\left(\tau_{k}\right)\right) \dot{\sigma}^{\mu}\left(\tau_{k}\right)\right) \ldots\left(-A_{\mu}\left(\sigma\left(\tau_{1}\right)\right) \dot{\sigma}^{\mu}\left(\tau_{1}\right)\right)
$$

где

$$
\Delta_{s, t}^{k}:=\left\{\left(\tau_{1}, \ldots, \tau_{k}\right) \in \mathbb{R}^{k}: s \leqslant \tau_{1} \leqslant \ldots \leqslant \tau_{k} \leqslant t\right\} .
$$

Оператор $U_{1,0}(\sigma)$ - параллельный перенос вдоль кривой $\sigma$.

Пусть функция $U: C^{1}\left([0,1], \mathbb{R}^{1,3}\right) \rightarrow M_{N}(\mathbb{C})$ определена формулой

$$
U(\sigma)=U_{1,0}(\sigma), \sigma \in C^{1}\left([0,1], \mathbb{R}^{1,3}\right) .
$$

В [17] доказывается, что функция $U$ (параллельный перенос вдоль $C^{1}$-гладких кривых) лежит в $\Omega$, причем $K_{\mu \nu}^{V}, K_{\mu \nu}^{L}, K_{\mu \nu}^{S}$ определяются так:

$$
\begin{aligned}
& K_{\mu \nu}^{V}(\sigma)(t, s)= \\
& \quad= \begin{cases}U_{1, t}(\sigma) F_{\mu \lambda}(\sigma(t)) \dot{\sigma}^{\lambda}(t) U_{t, s}(\sigma) F_{\nu \kappa}(\sigma(s)) \dot{\sigma}^{\kappa}(s) U_{s, 0}(\sigma), & \text { если } t \geqslant s \\
U_{1, s}(\sigma) F_{\nu \kappa}(\sigma(s)) \dot{\sigma}^{\kappa}(s) U_{s, t}(\sigma) F_{\mu \lambda}(\sigma(t)) \dot{\sigma}^{\lambda}(t) U_{t, 0}(\sigma), & \text { если } t<s,\end{cases}
\end{aligned}
$$

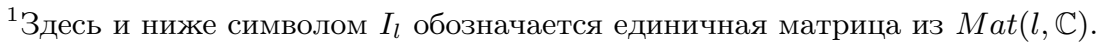




$$
\begin{gathered}
K_{\mu \nu}^{L}(\sigma)(t)=\frac{1}{2} U_{1, t}(\sigma)\left(-\nabla_{\mu} F_{\nu \lambda}(\sigma(t)) \dot{\sigma}^{\lambda}(t)-\nabla_{\nu} F_{\mu \lambda}(\sigma(t)) \dot{\sigma}^{\lambda}(t)\right) U_{t, 0}(\sigma), \\
K_{\mu \nu}^{S}(\sigma)(t)=\frac{1}{2} U_{1, t}(\sigma) F_{\mu \nu}(\sigma(t)) U_{t, 0}(\sigma) .
\end{gathered}
$$

Выполняется следующая теорема.

Теорема 2. Следующие два утверждения равносильны:

1) связность $A$ на $\mathbb{R}^{1,3}$ является решением уравнений Янга-Миллса:

$$
\nabla^{\mu} F_{\mu \nu}=0
$$

2) функиия $U$ является решением уравнения Даламбера-Леви:

$$
\square_{L_{1}} U(\sigma)=0
$$

Доказательство см. в [17].

В силу теоремы 1 в теореме 2 даламбертиан Леви, определенный с помощью интегрального представления, можно заменить на даламбертиан Леви в смысле среднего Чезаро вторых производных по направлению. Отсюда следует, что если параллельный перенос как функция на пространстве $C^{1}$-гладких кривых зависит от конечного количества элементов базиса, то соответствующая связность является решением уравнений Янга-Миллса.

Следующая теорема утверждает, что если параллельный перенос зависит от конечного количества элементов базиса, то соответствующая связность является плоской.

Теорема 3. Пусть $\left\{h_{n}\right\}$-ортонормированный базис в $L_{2}([0,1], \mathbb{R})$ такой, что все элементы $\left\{h_{n}\right\}$ принадлежсат $C^{1}([0,1], \mathbb{R})$, причем $h_{n}(0)=0$ для всех $n \in \mathbb{N}$ и $h_{n}(1)=0$ для всех $n>N_{1}$ для некоторого натурального $N_{1}$. Если для каждого $\sigma \in C^{1}\left([0,1], \mathbb{R}^{1,3}\right)$ выполняется

$$
d_{p_{\mu} h_{n}} U(\sigma)=0
$$

для всех $\mu$ и $n>N_{\sigma}$ для некоторого натурального $N_{\sigma}$, то $F \equiv 0$.

Доказ ат ельство. Известно (см. [17]), что

$$
\begin{aligned}
d_{u} U(\sigma)=\int_{0}^{1} U_{1, t}(\sigma)( & \left.-F_{\mu \nu}(\sigma(t)) u^{\mu}(t) \dot{\sigma}^{\nu}(t)\right) U_{t, 0}(\sigma) d t+ \\
& +U_{1,0}(\sigma) A_{\mu}(\sigma(1)) u^{\mu}(1)-A_{\mu}(\sigma(1)) u^{\mu}(1) U_{1,0}(\sigma) .
\end{aligned}
$$

Зафиксируем $\sigma \in C^{1}\left([0,1], \mathbb{R}^{1,3}\right)$. Пусть $N_{\sigma}^{1}=\max \left(N_{\sigma}, N_{1}\right)$. Введем функцию $J_{\mu}:[0,1] \rightarrow M_{N}(\mathbb{C})$, определенную формулой

$$
J_{\mu}(t)=U_{1, t}(\sigma)\left(-F_{\mu \nu}(\sigma(t)) \dot{\sigma}^{\nu}(t)\right) U_{t, 0}(\sigma) .
$$

Тогда в силу (12) для $n>N_{\sigma}^{1}$ выполняется

$$
d_{p_{\mu} h_{n}} U(\sigma)=\int_{0}^{1} J_{\mu}(t) h_{n}(t) d t=0 .
$$


Т.е. $\left(J_{\mu}(t)\right)_{j}^{i} \perp h_{n}$ в $L_{2}([0,1])$ для всех $n>N_{\sigma}^{1}$ и $i, j \in\{1, \ldots, N\}$. Тогда

$$
J_{\mu}(t)=\sum_{n=1}^{N_{\sigma}^{1}} M_{n} h_{n}(t)
$$

где

$$
M_{n}=\int_{0}^{1} J_{\mu}(t) h_{n}(t) d t
$$

Отсюда следует, что

$$
-U_{1,0}(\sigma) F_{\mu \nu}(\sigma(0)) \dot{\sigma}^{\nu}(0)=J_{\mu}(0)=\sum_{n=1}^{N_{\sigma}^{1}} M_{n} h_{n}(0)=0 .
$$

Тогда $F_{\mu \nu}(\sigma(0)) \dot{\sigma}^{\nu}(0)=0$ для всех $\sigma \in C^{1}\left([0,1], \mathbb{R}^{1,3}\right)$. Подбирая подходящие $\sigma \in C^{1}\left([0,1], \mathbb{R}^{1,3}\right)$, мы получаем $F \equiv 0$.

4. Даламбертиан Леви и уравнения квантовой хромодинамики. Пусть

$$
W_{2,0}^{1}([0,1], \mathbb{R})=\left\{\sigma \in W_{2}^{1}([0,1], \mathbb{R}): \sigma(0)=0\right\}
$$

- гильбертово пространство со скалярным произведением

$$
\left(\sigma_{1}, \sigma_{2}\right)_{H}=\int_{0}^{1} \dot{\sigma}_{1}(t) \dot{\sigma}_{2}(t) d t
$$

Функции $\left\{f_{n}\right\}_{n=0}^{\infty}$, где $f_{0}(t)=t$ и $f_{n}(t)=\frac{\sqrt{2}}{\pi n} \sin (\pi n t)$ для $n \in \mathbb{N}$, образуют ортонормированный базис $W_{2,0}^{1}([0,1], \mathbb{R})$. Пусть

$$
H=\left\{\sigma \in W_{2}^{1}\left([0,1], \mathbb{R}^{1,3}\right): \sigma(0)=0\right\}=\mathbb{R}^{1,3} \otimes W_{2,0}^{1}([0,1], \mathbb{R}) .
$$

Пусть функция $U_{w}: H \rightarrow M_{N}(\mathbb{C})$ определена формулой

$$
U_{w}(\sigma)=U_{1,0}(\sigma), \sigma \in H
$$

В дальнейшем мы будем рассматривать перенос вдоль кривых из $H$ и пользоваться даламбертианом Леви, определенным с помощью базиса $\left\{f_{n}\right\}_{n=0}^{\infty}$, т.к. с помощью этого базиса можно определить производную в конечной точке.

Следующее определение является частным случаем определения 1.

ОПРедЕЛЕНиЕ 4. Неклассический даламбертиан Леви $\square_{L_{2}}-$ это линейное отображение из пространства $\operatorname{dom} \square_{L_{2}}$ в пространство всех $M_{N}(\mathbb{C})$-значных функций на $H$, определенное следующим образом:

$$
\square_{L_{2}} f(\sigma)=\lim _{n \rightarrow \infty} \frac{1}{n} \sum_{k=1}^{n}\left(d_{\pi k p_{0} f_{k}} d_{\pi k p_{0} f_{k}} f(\sigma)-\sum_{\mu=1}^{3} d_{\pi k p_{\mu} f_{k}} d_{\pi k p_{\mu} f_{k}} f(\sigma)\right),
$$

где $\operatorname{dom} \square_{L_{2}}$ - пространство дважды дифференцируемых по Гато $M_{N}(\mathbb{C})$ значных функций на $H$, для которых правая часть (13) существует для всех $\sigma \in H$. 
В доказательстве теоремы 4 и ниже символом $\sigma^{r}(r \in[0,1])$ обозначается кривая из $H$, определенная так: $\sigma^{r}(t)=\sigma(r t)$.

Теорема 4. Пусть $J_{\nu}(x) d x^{\nu}-C^{\infty}$-гладкая $u(N)$-значная 1-форма. Следующие два утверждения равносильны:

1) связность А является решением уравнений Янга-Миллса с источником:

$$
\nabla^{\mu} F_{\mu \nu}=J_{\nu}
$$

2) для каждого $\sigma \in H$ выполняется соотношение

$$
\square_{L_{2}} U_{w}(\sigma)=-\int_{0}^{1} U_{1, t}(\sigma) J_{\nu}(\sigma(t)) \dot{\sigma}^{\nu}(t) U_{t, 0}(\sigma) d t
$$

До к а з а тел в с т в о. Функция $U_{w}$ бесконечно дифференцируема по Фреше на $H$ (см. [22]). Такими же рассуждениями, как и в [17], получаем, что для второй производной $U_{w}$ выполняется соотношение (6) для всех $\sigma, u, v \in H$ таких, что $u(1)=v(1)=0$, причем $K_{\mu \nu}^{V}, K_{\mu \nu}^{L}, K_{\mu \nu}^{S}$ выражаются формулами (8), (9), (10) соответственно.

Так как

$$
\lim _{n \rightarrow \infty} \frac{1}{n}\left(\sum_{k=1}^{n}(\pi k)^{2} f_{k}^{2}(t)\right)=1
$$

равномерно на каждом отрезке $\left[t_{1}, t_{2}\right] \in(0,1)$ и

$$
\sup _{t \in[0,1], n \in \mathbb{N}}\left|\frac{1}{n}\left(\sum_{k=1}^{n}(\pi k)^{2} f_{k}^{2}(t)\right)\right| \leqslant 2
$$

(см. [2]), по теореме Лебега

$$
\begin{aligned}
& \lim _{n \rightarrow \infty} \frac{1}{n} \sum_{k=1}^{n} \int_{0}^{1} K_{\mu \mu}^{L}(\sigma)(t)(\pi k)^{2} f_{k}^{2}(t) d t= \\
&=\lim _{n \rightarrow \infty} \frac{1}{n} \sum_{k=1}^{n} \int_{0}^{1} U_{1, t}(\sigma)\left(-\nabla_{\mu} F_{\mu \lambda}(\sigma(t)) \dot{\sigma}^{\lambda}(t)(\pi k)^{2} f_{k}^{2}(t)\right) U_{t, 0}(\sigma) d t= \\
& \quad=\int_{0}^{1} U_{1, t}(\sigma)\left(-\nabla_{\mu} F_{\mu \lambda}(\sigma(t)) \dot{\sigma}^{\lambda}(t)\right) U_{t, 0}(\sigma) d t
\end{aligned}
$$

для каждого $\mu \in\{0,1,2,3\}$. Тогда

$$
\square_{L_{2}} U_{w}(\sigma)=\int_{0}^{1} U_{1, t}(\sigma)\left(-\nabla^{\mu} F_{\mu \lambda}(\sigma(t)) \dot{\sigma}^{\lambda}(t)\right) U_{t, 0}(\sigma) d t .
$$

Отсюда очевидно следует, что если верно $\nabla^{\mu} F_{\mu \nu}=J_{\nu}$, то для параллельного переноса выполняется (14).

Докажем обратное утверждение. Для каждой $\sigma \in C^{1}\left([0,1], \mathbb{R}^{1,3}\right)$ введем функцию $R_{\sigma}$ на отрезке $[0,1]$ следующим образом:

$$
R_{\sigma}(r)=\int_{0}^{r} U_{1, t}(\sigma)\left(-\nabla^{\mu} F_{\mu \nu}(\sigma(t))+J_{\nu}(\sigma(t))\right) \dot{\sigma}^{\nu}(t) U_{t, 0}(\sigma) d t
$$


Taк как

$$
\dot{\sigma}^{r \nu}(t)=r \dot{\sigma}^{\nu}(r t), \quad U_{(r t),(r s)}(\sigma)=U_{t, s}\left(\sigma^{r}\right)
$$

выполняется

$$
R_{\sigma}(r)=U_{1, r}(\sigma)\left(\square_{L_{2}} U_{w}\left(\sigma^{r}\right)+\int_{0}^{1} U_{1, t}\left(\sigma^{r}\right) J_{\nu}\left(\sigma^{r}(t)\right) \dot{\sigma}^{r \nu}(t) U_{t, 0}\left(\sigma^{r}\right) d t\right) \equiv 0 .
$$

Тогда

$$
R_{\sigma}^{\prime}(r)=U_{1, r}(\sigma)\left(-\nabla^{\mu} F_{\mu \nu}(\sigma(r)) \dot{\sigma}^{\nu}(r)+J_{\nu}(\sigma(r)) \dot{\sigma}^{\nu}(r)\right) U_{r, 0}(\sigma) \equiv 0 .
$$

Так как $U_{1, r}(\sigma), U_{r, 0}(\sigma) \in U(N)$, мы получаем

$$
\nabla^{\mu} F_{\mu \nu}(\sigma(r)) \dot{\sigma}^{\nu}(r)=J_{\nu}(\sigma(r)) \dot{\sigma}^{\nu}(r)
$$

для всех $\sigma \in C^{1}\left([0,1], \mathbb{R}^{1,3}\right)$ и для всех $r \in[0,1]$. Тогда $\nabla^{\mu} F_{\mu \nu}=J_{\nu}$.

ОПредЕление 5 . Если функция $f: H \rightarrow V_{1}$, где $V_{1}-$ конечномерное векторное пространство, дифференцируема всюду по Гато, то производная $f$ в конечной точке по направлению $h \in \mathbb{R}^{1,3}$ (endpoint derivation) определяется формулой

$$
D_{h} f(\sigma)=d_{h f_{0}} f(\sigma)+\sum_{n=1}^{\infty} \sqrt{2}(-1)^{n} d_{h f_{n}} f(\sigma),
$$

если правая часть существует для всех $\sigma \in H$.

Будем обозначать $D_{\mu}=D_{p_{\mu}}$.

ПредложениЕ 1. Пусть $f: H \rightarrow V_{1}$, где $V_{1}$ - конечномерное векторное пространство, дифберениируема всюду на $Н$ в смысле Гато, причем производная $f$ представляется в виде

$$
d_{u} f(\sigma)=\int_{0}^{1}\left\langle u(t), \nu_{\sigma}(d t)\right\rangle
$$

где для каждого $\sigma \in H \nu_{\sigma}-э т о V_{1} \times\left(\mathbb{R}^{1,3}\right)^{*}$-значная борелевская мера. Тогда существует производная функиии $f$ в конечной точке по направлению $h \in \mathbb{R}^{1,3}$, причем

$$
D_{h} f(\sigma)=\left\langle h, \nu_{\sigma}(\{1\})\right\rangle, \quad \sigma \in H .
$$

Доказательство. Ряд Фурье

$$
\sum_{n=1}^{\infty}(-1)^{n} \frac{2}{\pi n} \sin \pi n t
$$

сходится к $t$ на $[0,1)$, причем частичные суммы этого ряда равномерно ограничены на $[0,1]$ (см. например [23, с. 555]). Тогда предложение следует из теоремы Лебега, т.к. ряд

$$
f_{0}(t)+\sum_{n=1}^{\infty}(-1)^{n} \sqrt{2} f_{n}(t)
$$


сходится поточечно к $\mathbf{1}_{\{1\}}(t)$ (индикатору точки $\{1\}$ ), причем частичные суммы ряда равномерно ограничены на $[0,1]$.

Пусть $\left\{g_{\alpha}\right\}_{\alpha=1}^{4}-$ фиксированный базис в $\mathbb{C}^{4}$. Если $\varphi \in \mathbb{C}^{N} \otimes \mathbb{C}^{4}$, символами $\varphi_{\alpha}(\alpha=1,2,3,4)$ будем обозначать такие векторы из $\mathbb{C}^{N}$, что

$$
\varphi=\sum_{\alpha=1}^{4} \varphi_{\alpha} \otimes g_{\alpha}
$$

Пусть $\gamma^{\mu}$ - матрицы Дирака. ${ }^{2}$ Символ $\bar{\varphi} \gamma_{\mu} \varphi$ пусть обозначает оператор, действующий в $\mathbb{C}^{N}$ и определенный формулой

$$
\bar{\varphi} \gamma_{\mu} \varphi=\sum_{\alpha=1}^{4}\left(\left(I_{N} \otimes \gamma_{0} \gamma_{\mu}\right) \varphi\right)_{\alpha} \otimes \varphi_{\alpha}^{*}
$$

Для того чтобы вывести систему уравнений, эквивалентную уравнениям Янга-Миллса-Дирака, напомним несколько известных фактов (см. [22]).

ПрЕДЛОЖЕНИЕ 2. Если $\varphi \in \mathbb{C}^{N}$, mo $i \bar{\varphi} \gamma_{\mu} \varphi \in u(N)$.

Доказательство. Пусть $\xi_{1}, \xi_{2} \in \mathbb{C}^{N}$, тогда

$$
\begin{gathered}
\left(\bar{\varphi} \gamma_{\mu} \varphi \xi_{1}, \xi_{2}\right)_{\mathbb{C}^{N}}=\left(\sum_{\alpha=1}^{4}\left(\left(I_{N} \otimes \gamma_{0} \gamma_{\mu}\right)\left(\sum_{\beta=1}^{4} \varphi_{\beta} \otimes g_{\beta}\right)\right)_{\alpha}\left(\xi_{1}, \varphi_{\alpha}\right)_{\mathbb{C}^{N}}, \xi_{2}\right)_{\mathbb{C}^{N}}= \\
=\left(\sum_{\alpha=1}^{4}\left(\sum_{\beta=1}^{4} \varphi_{\beta} \otimes\left(\gamma_{0} \gamma_{\mu} g_{\beta}\right)\right)_{\alpha}\left(\xi_{1}, \varphi_{\alpha}\right)_{\mathbb{C}^{N}}, \xi_{2}\right)_{\mathbb{C}^{N}}= \\
=\sum_{\alpha=1}^{4} \sum_{\beta=1}^{4}\left(\xi_{1}, \varphi_{\alpha}\right)_{\mathbb{C}^{N}}\left(\gamma_{0} \gamma_{\mu} g_{\beta}, g_{\alpha}\right)_{\mathbb{C}^{4}}\left(\varphi_{\beta}, \xi_{2}\right)_{\mathbb{C}^{N}}
\end{gathered}
$$

Аналогично доказывается, что

$$
\left(\xi_{1}, \bar{\varphi} \gamma_{\mu} \varphi \xi_{2}\right)_{\mathbb{C}^{N}}=\sum_{\alpha=1}^{4} \sum_{\beta=1}^{4}\left(\xi_{1}, \varphi_{\alpha}\right)_{\mathbb{C}^{N}}\left(g_{\beta}, \gamma_{0} \gamma_{\mu} g_{\alpha}\right)_{\mathbb{C}^{4}}\left(\varphi_{\beta}, \xi_{2}\right)_{\mathbb{C}^{N}}
$$

Так как $\gamma_{0} \gamma_{\mu}$ - симметричный оператор на $\mathbb{C}^{4}$ для каждого $\mu \in\{0,1,2,3\}$, выполняется $\left(\bar{\varphi} \gamma_{\mu} \varphi \xi_{1}, \xi_{2}\right)_{\mathbb{C}^{N}}=\left(\xi_{1}, \bar{\varphi} \gamma_{\mu} \varphi \xi_{2}\right)_{\mathbb{C}^{N}}$. Тогда $\left(i \bar{\varphi} \gamma_{\mu} \varphi\right)^{*}=-i \bar{\varphi} \gamma_{\mu} \varphi$.

ПрЕДЛОЖЕНИЕ 3. Если $g \in U(N) u \varphi \in \mathbb{C}^{N}$, mo

$$
\overline{\left(g \otimes I_{4}\right) \varphi} \gamma_{\mu}\left(g \otimes I_{4}\right) \varphi=g \bar{\varphi} \gamma_{\mu} \varphi g^{-1} .
$$

Доказательств о. Так как операторы $\left(g \otimes I_{4}\right)$ и $\left(I_{N} \otimes \gamma_{0} \gamma_{\mu}\right)$ коммутируют, верна цепочка равенств

\footnotetext{
${ }^{2}$ Мы считаем, что $\gamma^{\mu} \in \operatorname{Mat}(4, \mathbb{C})$, причем $\gamma^{\mu} \gamma^{\nu}+\gamma^{\nu} \gamma^{\mu}=2 \eta^{\mu \nu} I_{4}, \gamma_{0}^{*}=\gamma_{0}, \gamma_{\mu}^{*}=-\gamma_{\mu}$ при $\mu \neq 0$.
} 


$$
\begin{aligned}
\overline{\left(g \otimes I_{4}\right) \varphi} \gamma_{\mu}\left(g \otimes I_{4}\right) \varphi & =\sum_{\alpha=1}^{4}\left(\left(g \otimes I_{4}\right)\left(I_{N} \otimes \gamma_{0} \gamma_{\mu}\right) \varphi\right)_{\alpha} \otimes\left(\left(g \otimes I_{4}\right) \varphi\right)_{\alpha}^{*}= \\
= & \sum_{\alpha=1}^{4}\left(g\left(\left(I_{N} \otimes \gamma_{0} \gamma_{\mu}\right) \varphi\right)_{\alpha}\right) \otimes\left(g \varphi_{\alpha}\right)^{*}= \\
& =g\left(\sum_{\alpha=1}^{4}\left(\left(I_{N} \otimes \gamma_{0} \gamma_{\mu}\right) \varphi\right)_{\alpha} \otimes\left(\varphi_{\alpha}\right)^{*}\right) g^{-1}=g \bar{\varphi} \gamma_{\mu} \varphi g^{-1} .
\end{aligned}
$$

Предпоследнее равенство выполняется, т.к. для $\beta_{1}, \beta_{2} \in \mathbb{C}^{N}$ и $g \in U(N)$ верно равенство

$$
g\left(\beta_{1} \otimes \beta_{2}^{*}\right) g^{-1}=\left(\left(g \beta_{1}\right) \otimes\left(g \beta_{2}\right)^{*}\right) .
$$

Действительно, пусть $\xi \in \mathbb{C}^{N}$, тогда

$$
g\left(\beta_{1} \otimes \beta_{2}^{*}\right) g^{-1} \xi=g \beta_{1}\left(g^{-1} \xi, \beta_{2}\right)_{\mathbb{C}^{N}}=g \beta_{1}\left(\xi, g \beta_{2}\right)_{\mathbb{C}^{N}}=\left(\left(g \beta_{1}\right) \otimes\left(g \beta_{2}\right)^{*}\right) \xi .
$$

Tеорема 5. Пара $(A, \psi)$, где $\psi \in C^{\infty}\left(\mathbb{R}^{1,3}, \mathbb{C}^{N} \otimes \mathbb{C}^{4}\right)$, является решением системы уравнений квантовой хромодинамики

$$
\left\{\begin{array}{r}
\left(I_{N} \otimes \gamma^{\mu}\right)\left(\partial_{\mu}+A_{\mu} \otimes I_{4}\right) \psi+i m \psi=0 \\
\nabla^{\mu} F_{\mu \nu}=-i\left(\bar{\psi} \gamma_{\nu} \psi\right)
\end{array}\right.
$$

тогда и только тогда, когда параллельный перенос $U_{w}$ и функиия $\Psi: H \rightarrow$ $\mathbb{C}^{N} \otimes \mathbb{C}^{4}$, определенная так:

$$
\Psi(\sigma)=\left(U_{w}^{-1}(\sigma) \otimes I_{4}\right) \psi(\sigma(1)), \quad \sigma \in H
$$

являются решением системы

$$
\left\{\begin{array}{r}
\left(I_{N} \otimes \gamma^{\mu}\right) D_{\mu} \Psi+i m \Psi=0 \\
\square_{L_{1}} U_{w}(\sigma)=i U_{w}(\sigma) \int_{0}^{1} \overline{\Psi\left(\sigma^{r}\right)} \gamma_{\nu} \Psi\left(\sigma^{r}\right) \dot{\sigma}^{\nu}(r) d r
\end{array}\right.
$$

Доказательство. Для кривой $\sigma \in H$ оператор $U_{t, s}^{-1}(\sigma)$, где $0 \leqslant s \leqslant$ $t \leqslant 1$ является решением системы дифференциальных уравнений

$$
\left\{\begin{array}{r}
\frac{d}{d t} U_{t, s}^{-1}(\sigma)=U_{t, s}^{-1}(\sigma) A_{\mu}(\sigma(t)) \dot{\sigma}^{\mu}(t) \\
\frac{d}{d s} U_{t, s}^{-1}(\sigma)=-A_{\mu}(\sigma(s)) \dot{\sigma}^{\mu}(s) U_{t, s}^{-1}(\sigma) \\
\left.U_{t, s}^{-1}(\sigma)\right|_{t=s}=I_{N} .
\end{array}\right.
$$

Это решение выражается как ряд

$$
U_{t, s}^{-1}(\sigma)=I_{N}+\sum_{k=1}^{\infty} \int_{\Delta_{s, t}^{k}}\left(A_{\mu}\left(\sigma_{\tau_{1}}\right) \dot{\sigma}_{\tau_{1}}^{\mu}\right) \ldots\left(A_{\mu}\left(\sigma_{\tau_{k}}\right) \dot{\sigma}_{\tau_{k}}^{\mu}\right) d \tau_{1} \ldots d \tau_{k} .
$$


Тогда функция $U_{w}^{-1}$ дифференцируема всюду по Фреше. Аналогично (12) для любого $u \in H$ выполняется

$d_{u} U_{w}^{-1}(\sigma)=\int_{0}^{1} U_{r, 0}^{-1}(\sigma)\left(F_{\nu \mu}(\sigma(r)) \dot{\sigma}^{\mu}(r) u^{\nu}(r)\right) U_{1, r}^{-1}(\sigma) d r+U_{1,0}^{-1}(\sigma) A_{\mu}(\sigma(1)) u^{\mu}(1)$.

Тогда верна следующая цепочка равенств:

$$
\begin{aligned}
d_{u} \Psi(\sigma)= & \left(d_{u} U_{w}^{-1}(\sigma) \otimes I_{4}\right) \psi(\sigma(1))+\left(U_{w}^{-1}(\sigma) \otimes I_{4}\right) \partial_{u(1)} \psi(\sigma(1))= \\
= & \left(\int_{0}^{1} U_{r, 0}^{-1}(\sigma) F_{\mu \nu}(\sigma(r)) u^{\mu}(r) \dot{\sigma}^{\nu}(r) U_{r, 1}^{-1}(\sigma) d r \otimes I_{4}\right) \psi(\sigma(1))+ \\
& \quad+\left(U_{w}^{-1}(\sigma) A_{\mu}(\sigma(1)) \otimes I_{4}\right) u^{\mu}(1)+\left(U_{w}^{-1}(\sigma) \otimes I_{4}\right) \partial_{u(1)} \psi(\sigma(1)) .
\end{aligned}
$$

Отсюда следует, что производная по Гато функции $\Psi$ имеет вид (15). Тогда в силу предложения 1 выполняется

$$
D_{\mu} \Psi(\sigma)=\left(U_{w}^{-1}(\sigma) \otimes I_{4}\right)\left(\partial_{\mu} \psi(\sigma(1))+\left(A_{\mu}(\sigma(1)) \otimes I_{4}\right) \psi(\sigma(1)) .\right.
$$

Из последнего равенства следует, что пара $(A, \psi)$ удовлетворяет первому уравнению системы $(16)$ тогда и только тогда, когда пара $\left(U_{w}, \Psi\right)$ удовлетворяет первому уравнению системы (17).

По теореме 4 пара $(A, \psi)$ является решением второго уравнения системы (16) тогда и только тогда, когда для всех $\sigma \in H$ выполняется

$$
\square_{L_{1}} U_{w}(\sigma)=\int_{0}^{1} U_{1, r}(\sigma)\left(i \overline{\psi(\sigma(r))} \gamma_{\nu} \psi(\sigma(r)) \dot{\sigma}^{\nu}(r)\right) U_{r, 0}(\sigma) d r
$$

Из предложения 3 для $g=U_{r, 0}^{-1}(\sigma)$ следует, что

$$
\begin{gathered}
U_{1,0}(\sigma) \int_{0}^{1} U_{r, 0}^{-1}(\sigma) \overline{\psi(\sigma(r))} \gamma_{\nu} \psi(\sigma(r)) \dot{\sigma}^{\nu}(r) U_{r, 0}(\sigma) d r= \\
=U_{1,0}(\sigma) \int_{0}^{1} \overline{\Psi\left(\sigma^{r}\right)} \gamma_{\nu} \Psi\left(\sigma^{r}\right) \dot{\sigma}^{\nu}(r) d r .
\end{gathered}
$$

Отсюда следует, что $(A, \psi)$ удовлетворяют второму уравнению системы $(16)$ тогда и только тогда, когда $\left(U_{w}, \Psi\right)$ удовлетворяют второму уравнению системы (17).

Верна аналогичная теорема для даламбертиана Леви и производной в конечной точке, определенных с помощью интегральных представлений. В статье [22] Л. Гросса доказывалась эквивалентность первых уравнений систем из теоремы 5, при этом производная в конечной точке была определена с помощью интегрального представления.

Благодарности. Автор благодарит И. В. Воловича и О. Г. Смолянова за полезные обсуждения. Работа выполнена при поддержке гранта Правительства РФ для господдержки научных исследований, проводимых под руководством ведущих ученых, в ФГБОУ ВПО «Московский государственный университет имени М. В. Ломоносова» по договору № 11.G34.31.0054.

\section{ORCID}

Борис Олегович Волков: http://orcid.org/0000-0002-6430-9125 


\section{БИБЛИОГРАФИЧЕСКИЙ СПИСОК}

1. Волков Б. О. Даламбертианы Леви и их применение в квантовой теории / Четвертая международная конферениия «Математическая физика и ее приложения»: материалы конф.; ред. чл.-корр. РАН И. В. Волович; д.ф.-м.н., проф. В. П. Радченко. Самара: СамГТУ, 2014. С. 106-107.

2. Lévy P. Problèmes concrets d'analyse fonctionnelle. Paris: Gauthier-Villars, 1951. xiv +484 pp.

3. Feller M. N. The Lévy Laplacian/ Cambridge Tracts in Math.. vol.166. Cambridge: Cambridge Univ. Press, 2005.

4. Аккарди Л., Смолянов О. Г. Операторы Лапласа-Леви в пространствах функций на оснащенных гильбертовых пространствах // Матем. заметки, 2002. Т. 72, №1. С. 145150. doi : $10.4213 / \mathrm{mzm} 658$.

5. Аккарди Л., Смолянов О. Г. Формулы Фейнмана для эволюционных уравнений с лапласианом Леви на бесконечномерных многообразиях // Докл. РАН, 2006. Т. 407, № 5. C. $583-588$.

6. Аккарди Л., Смолянов О. Г. Классические и неклассические лапласианы Леви // Докл. PAH, 2007. Т. 417, № 1. С. 7-11.

7. Аккарди Л., Смолянов О. Г. Обобщенные лапласианы Леви и чезаровские средние // Докл. РАН, 2009. Т. 424, № 5. С. 583-587.

8. Accardi L., Ji U. C., Saitô K. Exotic Laplacians and Derivatives of White Noise // Infin. Dimens. Anal. Quantum. Probab. Relat. Top., 2011. vol.14, no.1. pp. 1-14. doi: 10.1142/ s0219025711004262.

9. Accardi L., Ji U. C., Saitô K. The Exotic (Higher Order Lévy) Laplacians Generate the Markov Processes Given by Distribution Derivatives of White Noise// Infin. Dimens. Anal. Quantum. Probab. Relat. Top., 2013. vol. 16, no.3, 1350020. 26 pp.. doi: 10.1142/ s0219025713500203.

10. Accardi L., Smolianov O. G. On Laplacians and traces // Conf. Semin. Univ. Bari, 1993. vol. 250. pp. 1-25.

11. Gomez F., Smolyanov O. G. Modified Lévy Laplacians // Russ. J. Math. Phys., 2008. vol. 15, no. 1. pp. 45-50. doi : 10.1134/s1061920808010056.

12. Kuo H.-H., Obata N., Saitô K. Lévy Laplacian of generalized functions on a nuclear space // Journal of Functional Analysis, 1990. vol. 94, no. 1. pp. 74-92. doi : 10.1016/0022-1236(90) 90028-j.

13. Saitô K. Infinite Dimensional Laplacians Associated with Derivatives of White Noise // Quantum Probability and Related Topics, 2013. vol.29. pp. 233-248. doi:10.1142/ 9789814447546_0015.

14. Volkov B. O. Lévy-Laplacian and the Gauge Fields // Infin. Dimens. Anal. Quantum. Probab. Relat. Top., 2012. vol. 15, no. 4, 1250027. 19 pp.. doi: 10.1142/s0219025712500270.

15. Volkov B. O. Quantum Probability and Lévy Laplacians // Russ. J. Math. Phys., 2013. vol. 20, no. 2. pp. 254-256. doi: 10.1134/s1061920813020118.

16. Volkov B. O. Hierarchy of Lévy-Laplacians and Quantum Stochastic Processes // Infin. Dimens. Anal. Quantum. Probab. Relat. Top., 2013. vol. 16, no. 4, 1350027. 20 pp.. doi : 10. 1142/s0219025713500276.

17. Accardi L., Gibilisco P., Volovich I. V. Yang-Mills gauge fields as harmonic functions for the Lévy-Laplacians // Russian J. Math. Phys., 1994. no. 2. pp. 235-250.

18. Accardi L., Gibilisco P., Volovich I. V. The Lévy Laplacian and the Yang-Mills equations // Rendiconti Lincei, 1993. vol.4, no. 3. pp. 201-206. doi: 10.1007/bf03001574.

19. Арефьева И. Я., Волович И. В. Функциональные высшие законы сохранения в калибровочных теориях / Обобщенные функиии и их применения в математической физике: Тр. Междунар. конф.. М.: ВЦ АН СССР, 1981. С. 43-49.

20. Léandre R., Volovich I. V. The Stochastic Lévy Laplacian and Yang-Mills equation on manifolds // Infin. Dimens. Anal. Quantum. Probab. Relat. Top., 2001. vol. 4, no. 2. pp. 161172. doi : 10.1142/s0219025701000449. 
21. Авербух В. И., Смолянов О. Г., Фомин С. В. Обобщенные функции и дифференциальные уравнения в линейных пространствах. II. Дифференциальные операторы и их преобразования Фурье / Тр. ММО, Т. 27. М.: Издательство Московского университета, 1972. C. 249-262.

22. Gross L. A Poincarè lemma for connection forms // Journal of Functional Analysis, 1985. vol. 63, no. 1. pp. 1-46. doi : 10.1016/0022-1236(85) 90096-5.

23. Фихтенгольц Г. М. Курс дифференциального и интегрального исчисления. Т. 3. М.: Физматлит, 2003. 728 с.

Поступила в редакцию 16/XII/2014;

в окончательном варианте - 13/III/2015;

принята в печать - 08/IV/2015.

Vestn. Samar. Gos. Techn. Un-ta. Ser. Fiz.-mat. nauki

[J. Samara State Tech. Univ., Ser. Phys. \& Math. Sci.], 2015, vol. 19, no. 2, pp. 241-258

ISSN: 2310-7081 (online), 1991-8615 (print)

doi: http://dx.doi.org/10.14498/vsgtu1372

\title{
MSC: $81 \mathrm{T13}$
}

\section{LÉVY D'ALAMBERTIANS AND THEIR APPLICATION IN THE QUANTUM THEORY*}

\section{B. O. Volkov}

N. E. Bauman Moscow State Technical University,

5/1, 2-ya Baumanskaya st., Moscow, 105005, Russian Federation.

\begin{abstract}
The Lévy d'Alambertian is the natural analogue of the well-known LévyLaplacian. The aim of the paper is the following. We study the relationship between different definitions of the Lévy d'Alambertian and the relationship between the Lévy d'Alambertian and the QCD equations (the Yang-MillsDirac equations). There are two different definitions of the classical Lévy d'Alambertian. One can define the Lévy d'Alambertian as an integral functional given by the second derivative or define it using the Cesaro means of the directional derivatives along the elements of some orthonormal basis. Using the weakly uniformly dense bases we prove the equivalence of these two definitions. We introduce the family of the nonclassical Lévy d'Alambertians using the family of the nonclassical Lévy Laplacians as a model. Any element of this family is associated with the linear operator on the linear span of the orthonormal basis. The classical Lévy d'Alambertian is an element of this family associated with the identity operator. We can describe the connection between the Lévy d'Alambertians and the gauge fields using the
\end{abstract}

(C) 2015 Samara State Technical University.

Please cite this article in press as:

Volkov B. O. Lévy d'Alambertians and their application in the quantum theory, Vestn. Samar. Gos. Tekhn. Univ., Ser. Fiz.-Mat. Nauki [J. Samara State Tech. Univ., Ser. Phys. \& Math. Sci.], 2015, vol. 19, no. 2, pp. 241-258. doi: 10.14498/vsgtu1372. (In Russian)

\section{Author Details:}

Boris O. Volkov (Cand. Phys. \& Math. Sci.; borisvolkov1986@gmail.com), Assistant Professor, Dept. of Mathematical simulation.

${ }^{*}$ This paper is an extended version of the paper [1], presented at the Mathematical Physics and Its Applications 2014 Conference. 
classical Lévy d'Alambertian or another nonclassical Lévy d'Alambertian specified in this paper. We study the relationship between this nonclassical Lévy d'Alambertian and the Yang-Mills equations with a source and obtain the system of infinite dimensional differential equations which is equivalent to the QCD equations.

Keywords: Lévy Laplacian, Lévy d'Alambertian, Yang-Mills equations, YangMills-Dirac equations.

doi: http://dx.doi.org/10.14498/vsgtu1372

Acknowledgments. The author is grateful to Igor V. Volovich and Oleg G. Smolyanov for helpful comments and insightful discussions.

The research was supported by the Grant of the Government of the Russian Federation for the support of scientific researches of the Government of the Russian Federation in the Federal State Budget Educational Institution of Higher Professional Education "Lomonosov Moscow State University" according to the agreement no. 11.G34.31.0054.

\section{ORCIDs}

Boris O. Volkov: http://orcid.org/0000-0002-6430-9125

\section{REFERENCES}

1. Volkov B. O. Lévy d'Alambertians and their application in the quantum theory, The 4nd International Conference "Mathematical Physics and its Applications", Book of Abstracts and Conference Materials; eds. I. V. Volovich; V. P. Radchenko. Samara, Samara State Technical Univ., 2014, pp. 106-107 (In Russian).

2. Lévy P. Problèmes concrets d'analyse fonctionnelle. Paris, Gauthier-Villars, 1951, xiv $+484 \mathrm{pp}$.

3. Feller M. N. The Lévy Laplacian, Cambridge Tracts in Math., vol.166. Cambridge, Cambridge Univ. Press, 2005.

4. Accardi L., Smolyanov O. G. Lévy-Laplace Operators in Functional Rigged Hilbert Spaces, Math. Notes, 2002, vol. 72, no. 1, pp. 129-134. doi: 10.1023/A:1019829424019.

5. Accardi L., Smolyanov O. G. Feynman formulas for evolution equations with Levy Laplacians on infinite-dimensional manifolds, Dokl. Math., 2006, vol. 73, no. 2, pp. 252-257. doi : 10.1134/s106456240602027x.

6. Accardi L., Smolyanov O. G. Classical and nonclassical Lévy Laplacians, Dokl. Math., 2007, vol. 76, no. 3, pp. 801-805. doi: 10.1134/s1064562407060014.

7. Accardi L., Smolyanov O. G. Generalized L'evy Laplacians and Cesâro means, Dokl. Math., 2009, vol. 79, no. 1, pp. 90-93. doi: 10.1134/S106456240901027X.

8. Accardi L., Ji U. C., Saitô K. Exotic Laplacians and Derivatives of White Noise, Infin. Dimens. Anal. Quantum. Probab. Relat. Top., 2011, vol.14, no.1, pp. 1-14. doi: 10.1142/ s0219025711004262.

9. Accardi L., Ji U. C., Saitô K. The Exotic (Higher Order Lévy) Laplacians Generate the Markov Processes Given by Distribution Derivatives of White Noise, Infin. Dimens. Anal. Quantum. Probab. Relat. Top., 2013, vol.16, no. 3, 1350020, 26 pp.. doi: 10.1142/ s0219025713500203.

10. Accardi L., Smolianov O. G. On Laplacians and traces, Conf. Semin. Univ. Bari, 1993, vol. 250, pp. 1-25.

11. Gomez F., Smolyanov O. G. Modified Lévy Laplacians, Russ. J. Math. Phys., 2008, vol. 15, no. 1, pp. 45-50. doi: 10.1134/s1061920808010056.

12. Kuo H.-H., Obata N., Saitô K. Lévy Laplacian of generalized functions on a nuclear space, Journal of Functional Analysis, 1990, vol. 94, no. 1, pp. 74-92. doi : 10.1016/0022-1236(90) 90028-j.

13. Saitô K. Infinite Dimensional Laplacians Associated with Derivatives of White Noise, Quantum Probability and Related Topics, 2013, vol.29, pp. 233-248. doi:10.1142/ 9789814447546_0015. 
14. Volkov B. O. Lévy-Laplacian and the Gauge Fields, Infin. Dimens. Anal. Quantum. Probab. Relat. Top., 2012, vol. 15, no. 4, 1250027, 19 pp.. doi: 10.1142/s0219025712500270.

15. Volkov B. O. Quantum Probability and Lévy Laplacians, Russ. J. Math. Phys., 2013, vol. 20, no. 2, pp. 254-256. doi: 10.1134/s1061920813020118.

16. Volkov B. O. Hierarchy of Lévy-Laplacians and Quantum Stochastic Processes, Infin. Dimens. Anal. Quantum. Probab. Relat. Top., 2013, vol. 16, no. 4, 1350027, 20 pp.. doi: 10. $1142 / \mathrm{s} 0219025713500276$.

17. Accardi L., Gibilisco P., Volovich I. V. Yang-Mills gauge fields as harmonic functions for the Lévy-Laplacians, Russian J. Math. Phys., 1994, no. 2, pp. 235-250.

18. Accardi L., Gibilisco P., Volovich I. V. The Lévy Laplacian and the Yang-Mills equations, Rendiconti Lincei, 1993, vol. 4, no. 3, pp. 201-206. doi: 10.1007/bf03001574.

19. Aref'eva I. Ya., Volovich I. V. Higher order functional conservation laws in gauge theories, Proc. Int. Conf. Generalized Functions and their Applications in Mathematical Physics. Moscow, Academy of Sciences of the USSR, 1981, 43-49 pp. (In Russian)

20. Léandre R., Volovich I. V. The Stochastic Lévy Laplacian and Yang-Mills equation on manifolds, Infin. Dimens. Anal. Quantum. Probab. Relat. Top., 2001, vol.4, no. 2, pp. 161172. doi : 10.1142/s0219025701000449.

21. Averbukh V. I., Smolyanov O. G., Fomin S. V. Generalized functions and differential equations in linear spaces. II. Differential operators and their Fourier transforms, Tr. Mosk. Mat. Obs., 27. Moscow, MSU, 1972, pp. 249-262 (In Russian).

22. Gross L. A Poincarè lemma for connection forms, Journal of Functional Analysis, 1985, vol. 63, no. 1, pp. 1-46. doi : 10.1016/0022-1236(85)90096-5.

23. Fikhtengolts G. M. Kurs differentsial'nogo i integral'nogo ischisleniia [Differential and integral calculus], vol. 3. Moscow, Fizmatlit, 2003, 728 pp. (In Russian)

Received 16/XII/2014;

received in revised form $13 / \mathrm{III} / 2015$;

accepted 08/IV/2015. 